features are mostly compatible with macrophage activation syndrome (MAS) observed in other viral infections despite resulting in milder changes. Early detection and treatment of MAS may be associated with a better outcome. However, available criteria for MAS associated with other causes have not been helpful. Objectives: To identify distinct features of MAS associated with COVID-19 using a large database enabling to assess of dynamic changes.

Methods: PCR-confirmed hospitalized COVID-19 patients followed between March and September 2020 constituted the discovery set. Patients considered to have findings of MAS by experienced physicians and given anakinra or tocilizumab were classified as the MAS group and the remaining patients as the non-MAS group. The MAS group was then re-grouped as the cases with exactMAS and borderline-MAS cases by the study group. Clinical and laboratory data including the $\mathrm{Ct}$ values of the PCR test were obtained from the database, and dynamic changes were evaluated especially for the first 14 days of the hospitalization. The second set of 162 patients followed between September-December 2020 were used as the replication group to test the preliminary criteria. In the second set, hospitalization rules were changed, and all patients required oxygen support and received dexamethasone $6 \mathrm{mg} /$ day or equivalent glucocorticoids. Daily changes were calculated for the laboratory items in MAS, borderline, and non-MAS groups to see the days differentiating the groups, and ROC curves and lower and upper limits (10-90\%) of the selected parameters were calculated to determine the cutoff values.

Results: A total of 769 PCR-confirmed hospitalized patients were analysed, and 77 of them were classified as MAS and 83 as borderline MAS patients. There was no statistically significant difference in the baseline viral loads of MAS patients compared to the non-MAS group according to the Ct values. Daily dynamic changes in the MAS group differed from the non-MAS group especially around the $6^{\text {th }}$ day of hospitalization, and more than a twofold increase in ferritin and a 1.5-fold increase in D-dimer levels compared to the baseline values help to define the MAS group. Twelve items selected for the criteria are given in Table 1 below. The total score of 45 provided $79.6 \%$ sensitivity for the MAS (including borderline cases) and $81.3 \%$ specificity around days 5 and 6 in the discovery set, and a score of 60 increased the specificity to $94.9 \%$ despite a decrease in sensitivity to $40.8 \%$. The same set provided a similar sensitivity $(80.3 \%)$ in the replication, but a lower specificity $(47.4-66 \%$ on days 6 to 9$)$ due to a group of control patients with findings of MAS possibly masked by glucocorticoids.

Table 1. Preliminary Criteria for Macrophage Activation Syndrome Associated with Coronavirus Disease-19

1. Fever $\left(>37.0^{\circ} \mathrm{C}\right)$

2.Ferritin concentration $>550 \mathrm{ng} / \mathrm{mL}$

3.More than 2 times increase of ferritin concentration within 7 days of disease onset

4.Neutrophil count $>6000 \mathrm{cell} / \mathrm{mm}^{3}$

5.Lymphopenia $<1000 \mathrm{cell} / \mathrm{mm}^{3}$

6. Neutrophil/lymphocyte ratio $>6$

7.D-dimer concentration $>1000 \mathrm{ng} / \mathrm{ml}$

8.More than $50 \%$ increase of $\mathrm{D}$-dimer concentration within 7 days of disease onse

9.CRP concetration $>50 \mathrm{mg} / \mathrm{L}$

10. $\mathrm{LDH}$ concentration $>300 \mathrm{U} / \mathrm{L}$

11.ALT or AST concentration $>50 \mathrm{U} / \mathrm{L}$

12.Procalcitonin concentration $<1.2$

1 point for each positive item assessed on Days 5-7Score calculation: Total points / $12 \mathrm{x}$ 100 Possible MAS $\geq 45$ and Definite MAS $\geq 60$

Conclusion: This study defined a set of preliminary criteria using the most relevant items of MAS according to the dynamic changes in the parameters in a group of COVID-19 patients. A score of 45 would be helpful to define a possible MAS group with reasonable sensitivity and specificity to start necessary treatments as early as possible.

Disclosure of Interests: None declared.

DOI: 10.1136/annrheumdis-2021-eular.3113

\section{OP0314 DOCK8 MUTATIONS IN COVID-19 AND MIS-C CYTOKINE STORM SYNDROME}

R. Cron ${ }^{1}$, M. Zhang ${ }^{1}$, D. Absher ${ }^{2}$, J. Bridges ${ }^{1}$, A. Schnell ${ }^{3}$, P. Bhatraju ${ }^{4}$, A. Vagrecha ${ }^{5}$, S. Lozinsky ${ }^{6}$, S. Acharya ${ }^{7}$, C. Levy ${ }^{7}$, W. Chatham ${ }^{3} .{ }^{1}$ University of Alabama at Birmingham, Pediatrics, Birmingham, United States of America; ${ }^{2}$ HudsonAlpha Institute for Biotechnology, Genomic Health Research, Huntsville, United States of America; ${ }^{3}$ University of Alabama at Birmingham, Medicine, Birmingham, United States of America; ${ }^{4}$ University of Washington, Medicine, Seattle, United States of America; ${ }^{5}$ Hofstra University, Pediatrics, Hempstead, United States of America; ${ }^{6}$ Hofstra University, Neurosurgery, Hampstead, United States of America; ${ }^{7}$ Hofstra University, Pediatrics, Hampstead, United States of America

Background: We recently identified DOCK8 as a novel gene associated with cytokine storm syndrome (CSS) ${ }^{1}$. Heterozygous missense mutations in DOCK 8 diminish NK cell lytic function and contribute to increased pro-inflammatory cytokine production (CSS). CSS is a potential complication of COVID-19 with severe consequences ${ }^{2}$. Children are at risk of a SARS-CoV-2 post-infectious CSS, multisystem inflammatory syndrome in children (MIS-C) ${ }^{3}$. Host genetic factors associated with COVID-19 CSS and MIS-C CSS are unknown.

Objectives: The goals are to identify and functionally study rare mutations in DOCK8 in patients with SARS-CoV-2 COVID-19 and MIS-C

Methods: To date, 16 adult patients enrolled in a COVID-19 CSS clinical trial at UAB had whole genome sequencing. Four $(25 \%)$ had rare heterozygous DOCK8 mutations (3 missense, 1 intronic). A COVID-19 CSS adult patient in Seattle also had a $D O C K 8$ missense mutation. In addition, DOCK 8 missense mutations were identified in five children (UAB \& Northwell) hospitalized with MIS-C. DOCK8 mutations, or wild-type (WT) sequence controls, were introduced into human NK-92 cells by FOAMY virus transduction. WT and mutant DOCK8-expressing NK-92 cells were incubated with K562 target cells and compared for cytolysis and degranulation (CD107a).

Results: One COVID-19 patient DOCK8 mutation (Gly523Arg) reduced NK cell degranulation by $30 \%$ and cytolysis by $23 \%(n=3)$ (Figure 1$)$. Similar studies of 3 MIS-C patients with DOCK8 missense mutations (Arg899Trp, Ala2Thr, Pro$687 \mathrm{Leu}$ ) revealed up to $31 \%$ reduced NK cell degranulation and $48 \%$ reduction in cytolysis by 3 distinct mutations $(n=3)$. Two-way ANOVA analysis revealed statistically significant $(p<0.05)$ differences in NK cell degranulation and lysis for fou unique DOCK8 mutations.
A

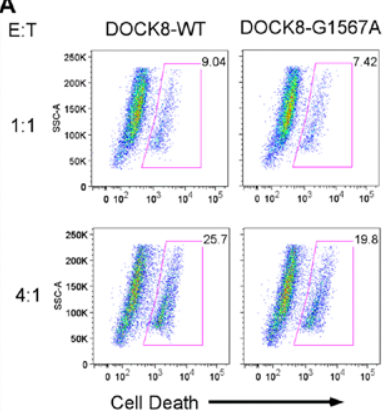

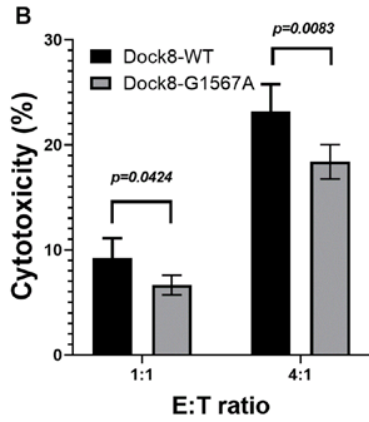

Conclusion: Heterozygous DOCK 8 missense mutations may contribute to severe COVID-19 and MIS-C CSS by partial dominant-negative effects yielding decreased NK cell cytolysis.

REFERENCES:

[1] Schulert GS, Cron RQ. The genetics of macrophage activation syndrome. Genes Immun 2020:21:169-181.

[2] Cron RQ, Chatham WW. The rheumatologist's role in COVID-19. J Rheumatol 2020:47:639-642.

[3] Reiff D, Mannion ML, Samuy N, Scalici P, Cron RQ. Distinguishing active pediatric COVID-19 from MIS-C. Pediatr Rheumatol Online J, in press.

Disclosure of Interests: Randy Cron Consultant of: SOBI, Novartis, Pfizer Sironax, Grant/research support from: SOBI, Mingce Zhang: None declared, Remy Cron: None declared, Devin Absher: None declared, John Bridges: None declared, Amanda Schnell: None declared, Pavan Bhatraju: None declared, Anshul Vagrecha: None declared, Shannon Lozinsky: None declared, Suchitra Acharya: None declared, Carolyn Levy: None declared, Winn Chatham Grant research support from: SOBI.

DOI: 10.1136/annrheumdis-2021-eular.1865

\section{Metabolic pathways during the regulation of inflam- mation and immunity}

\section{\begin{tabular}{|l|l}
\hline OP0315 & EFFECTOR CD4 T CELLS REQUIRE SURVIVIN FOR
\end{tabular} REGULATION OF GLUCOSE METABOLISM AND IFNG PRODUCTION}

M. Erlandsson ${ }^{1,2}$, K. M. Andersson ${ }^{1}$, N. Oparina ${ }^{1}$, S. Töyrä Silfverswärd ${ }^{1}$, M. I. Bokarewa ${ }^{1,2} .{ }^{1}$ Gothenburg University, Rheumatology and Inflammation Research, Gothenburg, Sweden; ${ }^{2}$ Sahlgrenska University Hospital, Rheumatology clinic, Göteborg, Sweden

Background: Interferon-gamma (IFNg) producing effector T cells play the leading role in triggering and perpetuation of inflammation in rheumatoid arthritis. Inflammation leads to metabolic reprogramming of T cells and high energy consumption supporting proliferation and IFNg production. Being a part of chromosomal passenger complex, oncoprotein survivin is essential for cell proliferation It has also been identified as a marker of severe therapy-resistant rheumatoid 
arthritis. Thus, we aimed to explore the association between survivin and IFNg producing phenotype of CD4 T cells.

Objectives: We study if survivin mediates the glucose dependent mechanism of IFNg production in CD4 T cells.

Methods: CD4 cells were sorted from the peripheral blood of RA patients and healthy controls, activated with aCD3, cultured in presence of survivin inhibitor YM155 and subjected to RNA sequencing (Illumina, Life Science). IFNg levels in supernatants were measured by ELISA. To study glucose uptake in presence of YM155, CD4 cells were treated with IFNg+aCD3 overnight followed by 2NBD-glucose challenge for $30 \mathrm{~min}$. Uptake of fluorescent 2NBD-glucose probe was measured by flow cytometry. Statistical analysis of RNAseq was performed in R-studio using the Bioconductor package DESeq2.

Results: Comparison of the whole-genome transcription profile of CD4 cells different by levels of BIRC5, coding for survivin, demonstrated that the BIRC5hi group expressed significantly higher levels of IFNg ( $\mathrm{mRNA}, \mathrm{p}=10^{-26}$ and protein, $\mathrm{p}=10^{-4}$ ). Also, BIRC5hi CD4 cells had higher expression of glucose transporter GLUT1 (SLC2A1, $p=0.0064$ ) and of glycolytic enzymes glucose-6-phosphate dehydrogenase (G6PD, $p=10^{-6}$ ), pyruvate kinase (PFKP, $p=10^{-6}$ ), and lactate dehydrogenase ( $\left.\mathrm{LDHA}, \mathrm{p}=10^{-14}\right)$. On the contrary, expression of the key regulator of glycolysis 6-phosphofructo-2-kinase (PFKFB3) was significantly lower in the BIRC5hi group $\left(p=4.4 \times 10^{-5}\right)$. Notably, expression of glycolytic enzymes G6PD and PFKFB3 correlated strongly to IFNg ( $r=0.880$ and -0.698 , respectively), TBX21 ( $r=0.811$ and -0.698$)$ and perforin $(r=0.781$ and -0.698$)$. To demonstrate functional relevance of the connection between BIRC5 and glucose metabolism, survivin was inhibited in CD4 cell cultures. Survivin inhibition resulted in significant increase of PFKFB3 $\left(p=7 \times 10^{-6}\right)$ and LDHA $(p=0.0089)$, leading to inhibition of phosphoglycerate mutase PGAM1 and ATF citrate lyase ACLY ( $p=0.021$ and $p=0.0074$, respectively), which dignify the restoration of aerobic glycolysis. Importantly, inhibition of survivin decreased 2NBD-glucose uptake by CD4 cells $(p=0.031)$ and reduced expression of GLUT1 $(p=0.034)$. These changes in glucose metabolism were followed by decreased IFNg production in supernatants $(\mathrm{p}=0.037)$.

Conclusion: The study demonstrates a strong connection between IFNg production and glucose metabolism in CD4 cells. Survivin emerges as an important regulator of glycolysis acting through expression of glycolytic enzymes and glucose transport

Disclosure of Interests: None declared.

DOI: 10.1136/annrheumdis-2021-eular.2295

\section{Let's work. Together.}

\begin{tabular}{|l|l}
\hline OP0316 & ASSOCIATION BETWEEN INDIVIDUAL AND \\
COUNTRY-LEVEL SOCIOECONOMIC FACTORS AND \\
WORK PARTICIPATION IN PERIPHERAL AND AXIAL \\
SPONDYLOARTHRITIS: ANALYSIS OF THE ASAS \\
PERSPA STUDY
\end{tabular}

S. S. Zhao ${ }^{1}$, E. Nikiphorou ${ }^{2}$, A. Boonen ${ }^{3,4}$, C. López-Medina ${ }^{5,6}$, M. Dougados ${ }^{5,7}$, S. Ramiro ${ }^{8,9}$. 'University of Liverpool, Musculoskeletal Biology, Institute of Life Course and Medical Sciences, Liverpool, United Kingdom $;{ }^{2}$ King's College London, Department of Rheumatology and Centre for Rheumatic Diseases, London, United Kingdom; ${ }^{3}$ Maastricht University, Care and Public Health Research Institute (CAPHRI), Maastricht, Netherlands; ${ }^{4}$ Academic Hospital Maastricht, Department of Internal Medicine, Division of Rheumatology, Maastricht, Netherlands; ${ }^{5}$ Cochin Hospital, Université de Paris, Rheumatology Department, Paris, France; ${ }^{6}$ University of Cordoba, Rheumatology Department, Reina Sofia Hospital, IMIBIC, Córdoba, Spain; ${ }^{7}$ University of Paris, INSERM (U1153): Clinical Epidemiology and Biostatistics, Paris, France; ${ }^{8}$ Leiden University Medical Center (LUMC), Department of Rheumatology, Leiden, Netherlands; ${ }^{9}$ Zuyderland MC, Department of Rheumatology, Heerlen, Netherlands

Background: Work outcomes in spondyloarthritis ( $\mathrm{SpA}$ ) have mostly been studied in axSpA, less in peripheral SpA (pSpA) or PsA. The ASAS-COMOSPA study showed that lower education, female gender and lower healthcare expenditures (HCE) are associated with lower odds of employment in SpA. However, it is unknown whether country-level factors or SpA phenotype (axSpA/pSpA/PsA) modify the effect of individual-level socioeconomic factors.

Objectives: To examine whether associations between socioeconomic factors and work outcomes differ across SpA phenotype and whether associations for individual-level socioeconomic factors are modified by country-level factors. Methods: Working age patients (18-65 years) from the ASAS-perSpA (peripheral involvement in $\mathrm{SpA}$ ) study were included. Associations between individual- (age, gender, education, marital status) and country-level socioeconomic factors (Human Development Index (HDI), HCE) with work outcomes (employment status (binary), absenteeism, presenteeism (tertiles)) were assessed using mixed-effects logistic and ordinal logistic models. Models were adjusted for confounders. Separate models for ASDAS, BASFI and BASDAI were created in turn due to collinearity. Effect modification by SpA phenotype and country-level factors was tested using interaction terms.

Results: A total of 3835 patients (mean age 42 years, $61 \%$ males) from 23 countries worldwide were included (66\% axSpA, 10\% pSpA, 23\% PsA). Being employed was associated with gender (male vs female OR $2.5 ; 95 \% \mathrm{Cl} 1.9-3.2$ ) education (university vs primary OR $3.7 ; 2.9-4.7$ ) and being married (vs single OR 1.3; 1.04-1.6) (Table 1). University (vs primary) education was associated with lower odds of absenteeism (OR 0.7; 0.5-0.7) and presenteeism (OR 0.5; 0.3-0.7) Associations were not statistically different across SpA phenotypes. HCE was significantly associated with all work outcomes: employment (OR 2.5; 1.5-4.1), absenteeism (OR 0.6; 0.4-0.9) and presenteeism (OR 0.6; 0.3-0.9). HDI results were similar. Gender discrepancy in odds of employment was greater in countries with lower socioeconomic development; eg, males had 3.5 higher odds of employment than females in countries with low HCE, whereas the difference was 1.8 fold in high HCE countries.

Table 1. Effect of individual socio-economic factors on work outcomes.

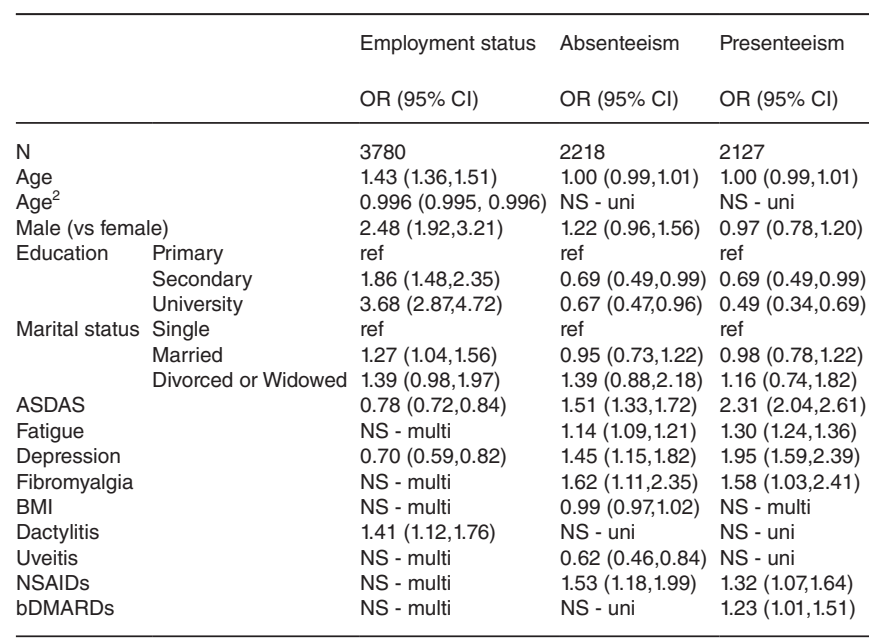

Models used ASDAS rather than BASDAI/BASFI, which are collinear. NS, not significant in the univariable or multivariable model.

Conclusion: Individual- (lower education) and country-level socioeconomic factors (lower healthcare expenditure) were both associated with (lower) work participation, independently of SpA phenotype. The disadvantageous effect of female gender on employment is particularly strong in countries with lower socioeconomic development. This highlights the need for wider societal interven tions, such as improving education and healthcare investment, to improve work outcomes.

Disclosure of Interests: Sizheng Steven Zhao: None declared, Elena Nikiphorou Speakers bureau: Pfizer, Lilly, AbbVie, Annelies Boonen Consultant of: Yes, Grant/research support from: Yes, Clementina López-Medina: None declared, Maxime Dougados: None declared, Sofia Ramiro Speakers bureau: Lilly, MSD, Novartis, UCB, Consultant of: AbbVie, Lilly, MSD, Novartis, UCB, Sanofi, Grant/ research support from: MSD.

DOI: 10.1136/annrheumdis-2021-eular.486

\section{OP0317-PARE THE CONTEMPORARY WORK-RELATED BURDEN OF DISEASE FOR PEOPLE WITH RHEUMATIC DISEASES IN GREECE}

K. Koutsogianni ${ }^{1}$, F. Asimakopoulou ${ }^{2}$, E. Repa ${ }^{3}$, I. Papadakis ${ }^{4}$,

M. Chatziioannou ${ }^{5}$, A. Verikiou ${ }^{2}$, M. Chatzigeorgiou $^{6}$, P. Pratsidou-Gertsi ${ }^{7}$, I. Chatzikrystallis ${ }^{8} .{ }^{1}$ PanHellenic Federation ReuMAzein, PanHellenic Federation ReuMAzein, Heraklion Crete, Greece; ${ }^{2}$ PanHellenic Federation ReuMAzein, Patients' Association with Rheumatic Diseases of Patra, Patra, Greece; 'PanHellenic Federation ReuMAZein, Parents' and Caregivers' Association of Children With Chronic Rheumatic Diseases, Thessaloniki, Greece; ${ }^{4}$ PanHellenic Federation ReuMAzein, The arthritis foundation of Crete, Heraklion Crete, Greece; ${ }^{5}$ PanHellenic Federation ReuMAZein, The arthritis foundation of Crete, Heraklion Crete, Greece; ${ }^{6}$ PanHellenic Federation ReuMAzein, PanHellenic Society of Patients with Autoimmune Rheumatic Diseases, Thessaloniki, Greece; ${ }^{7}$ PanHellenic Federation ReuMAZein, Friends Association of Children With Chronic Rheumatic Diseases, Thessaloniki, Greece; ${ }^{8}$ PanHellenic Federation ReuMAZein, PanHellenic Federation ReuMAZein, Thessaloniki, Greece 